\title{
Stress relaxation in ferroelectric materials
}

\author{
V C S PRASAD \\ Materials Development Laboratory, Bharat Electronics Limited, Bangalore 560013 , India \\ MS received 25 March 1982; revised 24 July 1982
}

\begin{abstract}
Stress relaxation takes place in $\mathrm{BaTiO}_{3}$ type ferroelectric materials due to the motion of non $180^{\circ}$ domain boundaries, which are the twin boundaries. The nature of stress relaxation taking place due to plastic deformation in crystalline solids and that due to twin boundaries in ferro electric materials is discussed. The usefulness of the stress relaxation data for the study of domain wall motions in this type of ferroelectrics is pointed out.
\end{abstract}

Keywords. Stress relaxation; ferroelectric domains; thermally activated deformation; ageing.

\section{Introduction}

Stress relaxation is a method to study the time-dependent deformation in solids. It has been widely used to study dislocation motions in plastically-deformed solids (Guiu and Pratt 1964; MacEwen et al 1969, Llyod and Embury 1971; Lee and Hart 1971). Timedependent deformation under constant load (creep) takes place in ferroelectric materials such as $\mathrm{BaTiO}_{3}$, due to the motion of the domain boundaries (Subbarao et al 1957; Syrkin and El'gard 1965). This time-dependent deformation also results in stress relaxation (Prasad and Subbarao 1973, 1977; Esaklul et al 1980). The present paper attempts to distinguish the type of information that can be obtained by stress relaxation studies in plastically-deformed solids and in ferroelectric materials, and examine the usefulness of this information to study the domain wall motions (twin boundaries) in ferroelectric materials.

\section{Stress relaxation in plastically deformed solids}

When a solid is plastically deformed in a testing machine and the motion of the crosshead arrested, stress relaxation takes place due to the motion of free dislocations. The decrease in load with time in such a case generally follows a logarithmic dependence. This test can be used to derive information on different aspects of the deformation process such as the activation energy and activation volume associated with the plastic deformation (Guiu and Pratt 1964), stress-strain rate dependence (Lee and Hart 1971), estimation of the thermal and athermal components of the flow stress (MacEwen et al 1969, Lloyd and Embury 1971) and information regarding the parameters controlling the dynamics of dislocation motion (Guiu and Pratt 1964).

In a stress-relaxation test, when the motion of the cross-head is arrested, the specimen continues to deform plastically at a decreasing rate under the action of a decreasing applied stress. In such a case, the plastic strain rate $\left(\dot{\varepsilon}^{*}\right)$ is related (Guiu and Pratt 1964) 
to the stress relaxation rate $\left(\dot{\sigma}^{*}\right)$ as

$$
\dot{\varepsilon}_{p}^{*}=-\left(\dot{\sigma}^{*} / M_{U}\right)
$$

where $M_{U}$ is the unrelaxed modulus. The plastic strain rate in terms of thermally activated plastic flow may also be given as

$$
\dot{\varepsilon}_{p}^{*}=A \exp -\left[Q-V \tau^{*} / R T\right]
$$

where $\dot{\varepsilon}_{p}^{*}$ is the plastic strain rate; $A$ is a constant which contains the entropy term; $Q$ is the activation energy of the deformation process $\ddagger$; $V$ the activation volume of the deformation process; $\tau^{*}$ is the shear stress or the flow stress; $R$, the gas constant; and $T$, the temperature in ${ }^{\circ} \mathrm{K}$.

Using eq. (1), eq. (2) can be integrated after converting the shear stress to the applied stress to obtain a relation between $\sigma^{*}$ and $t$ which is of the general form

$$
\sigma^{*}=K_{1}-K_{2} \log (t+c)
$$

where $K_{1}$ and $K_{2}$ are terms related to the activation volume, and $C$ is a constant. Hence a plot of $0^{*} v s \log t$ obtained from the stress relaxation data gives a straight line from which the activation volume of the deformation process can be estimated (Guiu and Pratt 1964).

\section{Stress relaxation in ferroelectric materials}

Stress relaxation in these materials takes place almost at negligible stresses, e.g. at approximately $0.1 \mathrm{~kg} / \mathrm{mm}^{2}$ in the single crystal $\mathrm{BaTiO}_{3}$ (Prasad and Subbarao 1973, 1977). The relaxation is due to the movement of twin boundaries which are the $90^{\circ}$ domain walls in $\mathrm{BaTiO}_{3}$. In single crystals, the relaxation takes place only in the region of deformation associated with twin boundary motions. In this region, the relaxation rate passes through a maximum (figure 1) with increasing stress (Prasad and Subbarao 1977). This relaxation depends on the stress, strain rate and temperature and hence can be treated as a thermally-activated process similar to dislocation motions in plastically-deformed solids (Esaklul et al 1980). However, unlike in plastic deformation, the material exhibits strong recovery effects (figure 2). Also, at low values of initial stress, the plot of the stress vs log time (figure 3) is convex towards the time axis whereas it tends to be concave with increase in stress (Prasad 1973). Similar relaxation behaviour was also observed in orthorhombic $\mathrm{KNbO}_{3}$ single crystals due to the motion of $60^{\circ}$ domain walls (Prasad and Subbarao 1972; Prasad 1973). Thus, it appears, that the above described relaxation behaviour is typical of polysynthetically-twinned crystals, the twinning taking place due to a single-twin system. Therefore qualitatively a similar relaxation behaviour might be expected in other systems such as In-Tl, Au-Cd, etc, which deform by twin boundary motions. Table 1 shows different twin systems and their associated Burgers vectors for twin dislocations in some ferroelectric materials known to show stress relaxation.

\footnotetext{
*indicates the thermal component of the particular parameter. For eg. $\sigma^{*}$ is the thermal component of the stress (or the effective stress) which is different from the long range internal stress.

$\$$ Artivation enthalpy $H$ is more often used by metallurgists in this expression although activation energy $Q$ was used by Esaklul et al (1980) who were the first to apply themall'-artivated deformation analysis to ferroelectric materials.
} 


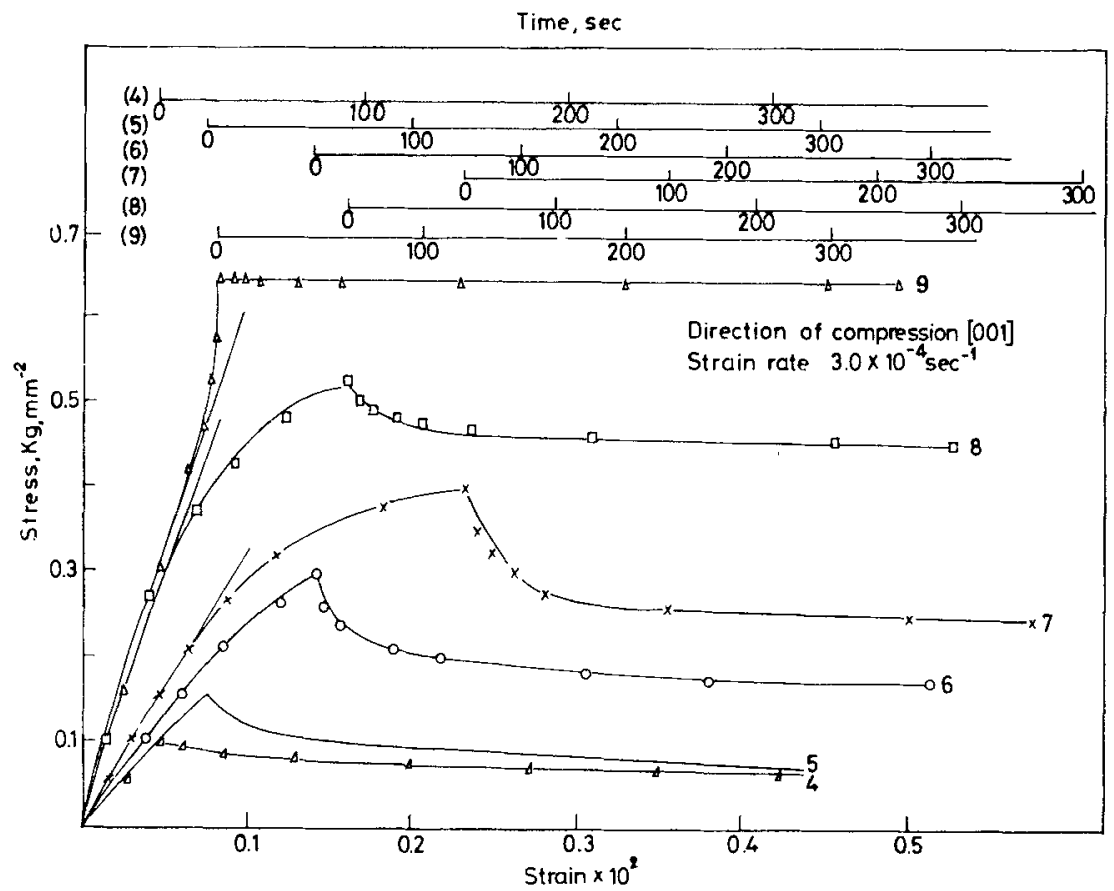

Figure 1. The stress relaxation behaviour of $\mathrm{BaTiO}_{3}$ single crystal with increasing stress (due to increasing deformation) $4.0 .11 \mathrm{~kg} \mathrm{~mm}^{-2} 5.0 .16 \mathrm{~kg} \mathrm{~mm}^{-2} 6.0 .3 \mathrm{~kg} \mathrm{~mm}^{-2} 7.0 .41 \mathrm{~kg} \mathrm{~mm}^{-2}$ 8. $0.53 \mathrm{~kg} \mathrm{~mm}^{-2}$ 9. $0.65 \mathrm{~kg} \mathrm{~mm}^{-2}$. (after Prasad and Subbarao 1977).

Table 1. I'win systems and Burgers vectors in some ferroelectric materials known to show stress relaxation.

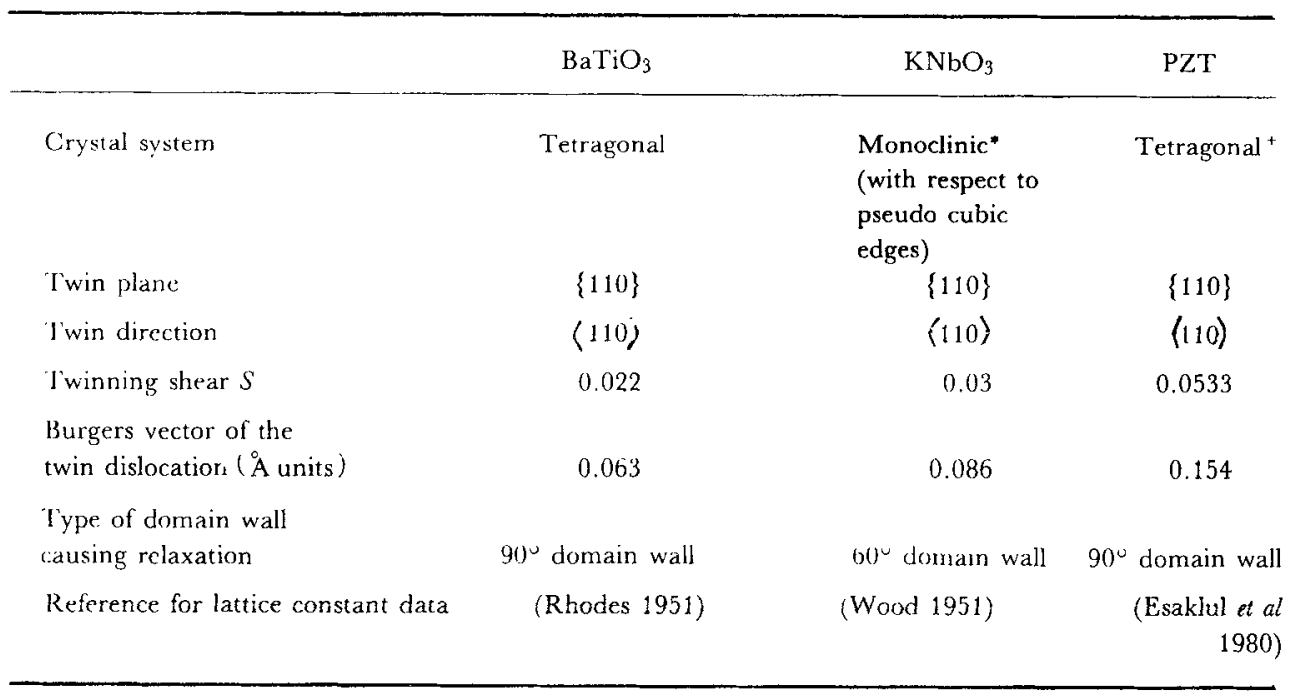

*'The mono linic angle between $a_{m}$ and $c_{m}$ axis (the psucdocubic edges of the untt cell) is $90^{\circ} 21$ '. This angle is assumed to be $90^{\circ}$ in estimating the Burgers vector.

+ This material may also exist in rhombohedral form at room temperature (Jaffe et al 1971). 


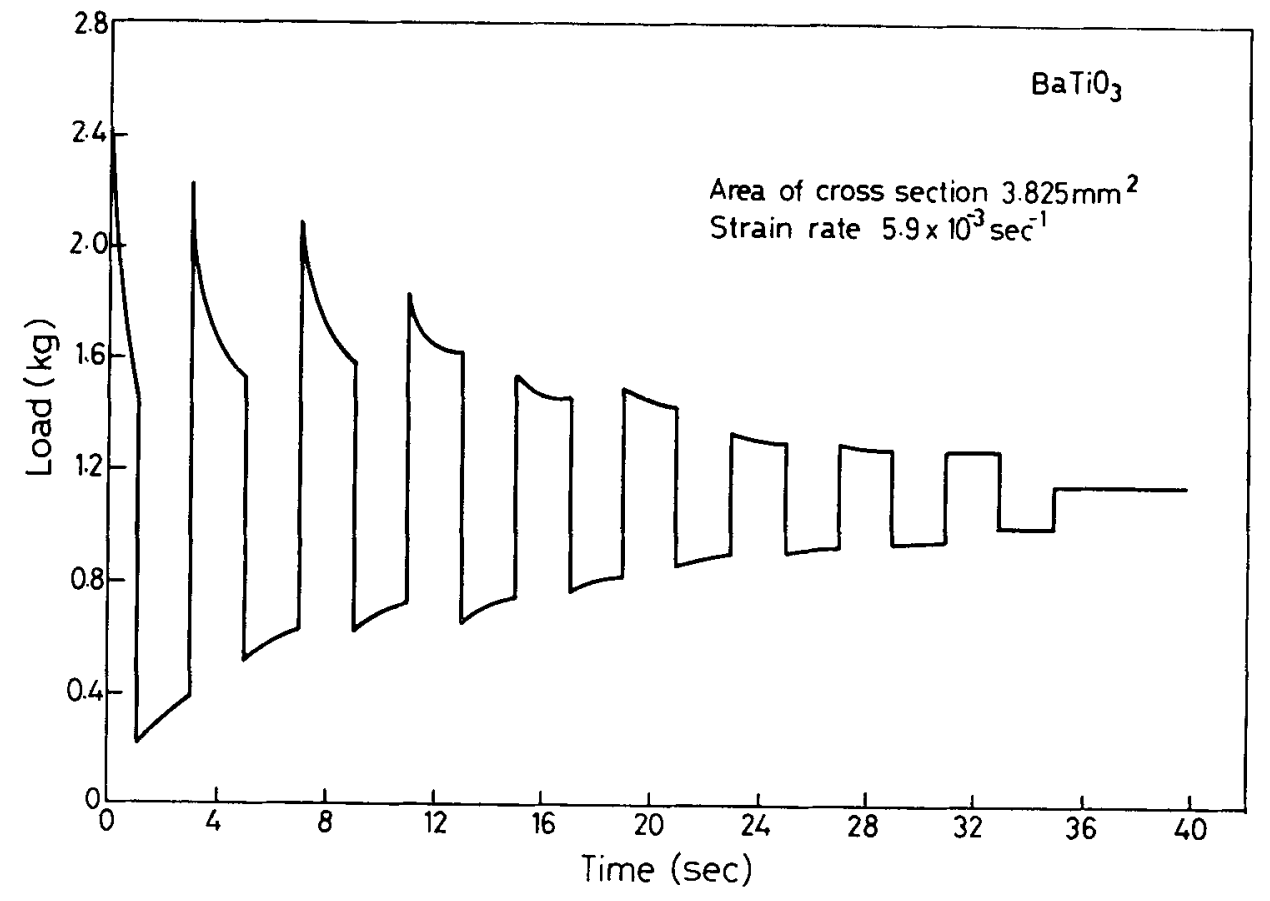

Figure 2. Relaxation and recovery behaviour of a $\mathrm{BaTiO}_{3}$ single crystal.

The above described features of relaxation are obviously due to anelastic deformation associated with twin boundary movements (ferro-electric domain walls) and is different from the plastic deformation due to dislocation motions that was discussed earlier. Such a relaxation behaviour was recently analyzed by Esaklul et al (1980) using Reed Hill and Dahlberg's analysis (1966) for thermally-activated deformation in anelastic materials. The main point of difference in this analysis from that the due to plastic deformation arising due to dislocation motions is the introduction of a term which contributes to reverse activation also, in (2) for thermally-activated deformation. In such a case, the relaxable strain rate as given in (2) takes the form (Reed Hill and Dahlberg 1966)

$$
\dot{\varepsilon}_{p}^{*}=A \exp -\left(Q-V \tau^{*}\right) / R T-\exp -\left(Q+V \tau^{*}\right) / R T
$$

Substituting for $\dot{\varepsilon}^{*}$ from (1) after converting the shear stress to the applied stress and the above equation can be integrated with respect to time gives the following equation (Esaklul et al 1980).

$$
\log \tan \mathrm{h}\left(\beta \sigma^{*} / 2 R T\right)-\left(t / \tau_{\varepsilon}\right)+C
$$

where $C$ is a constant of integration $t$ the time and $\tau_{\varepsilon}$ the relaxation time, defined by

$$
1 / \tau_{\varepsilon}=\left(2 M_{U} \beta / R T\right) A \exp -(Q / R T)
$$

where $\beta$ is directly proportional to the activation volume $V$, and $M_{U}$ is the unrelaxed modulus.

From these equations, the activation energy and the activation volume associated with the relaxation process can be estimated as shown by Esaklul et al (1980). From 


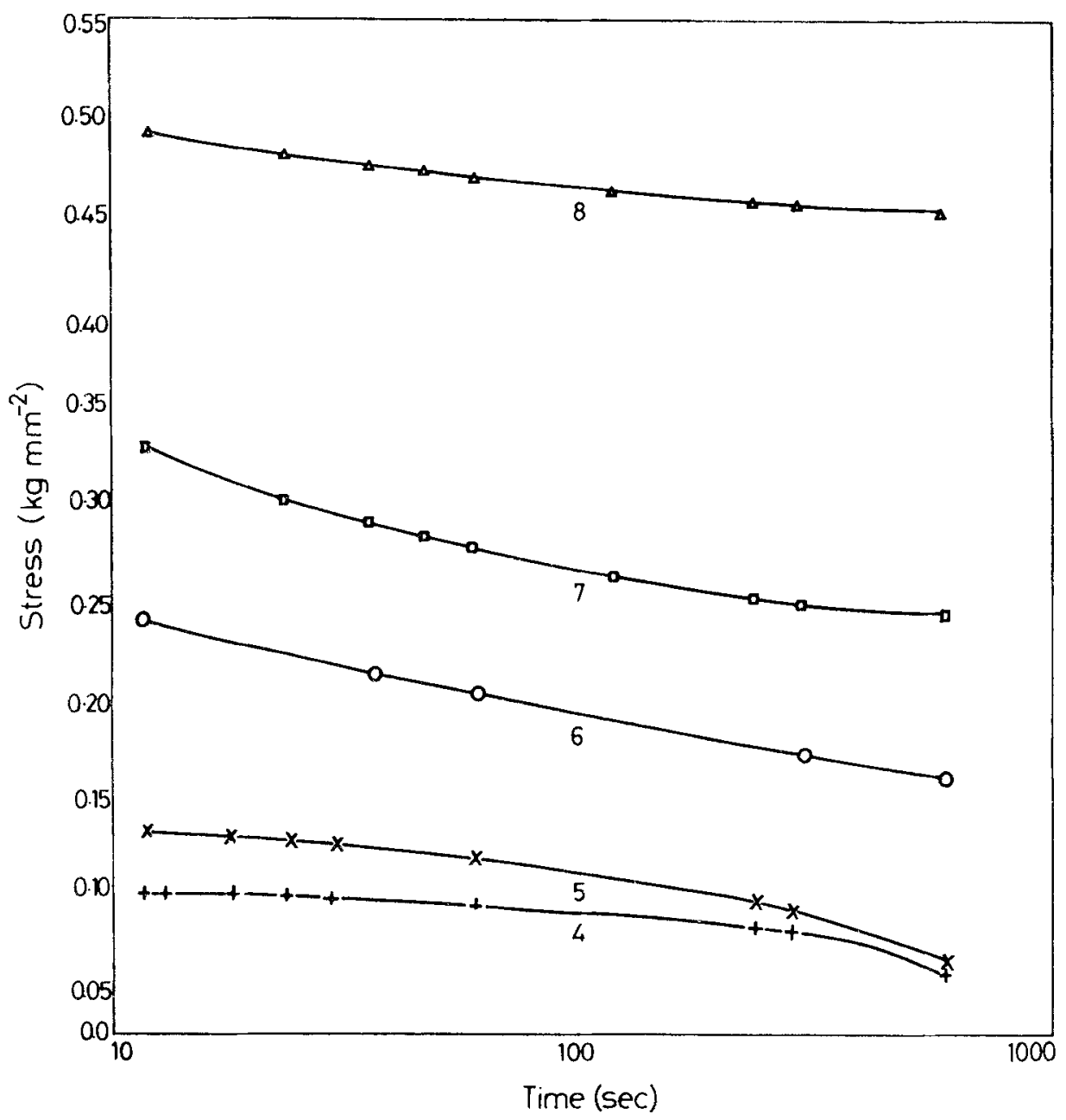

Figure 3. lime dependence of stress relaxation tor different initial stresses for a $\mathrm{BaTiO}_{3}$ single crystal.

such an analysis, an activation energy of $25 \mathrm{kcal} / \mathrm{mol}$ was obtained for the motion of boundaries in ceramic Lead Zirconate titanate (PZT). This activation energy was interpreted as the energy necessary to homogenously nucleate a twinning dislocation loop adjacent to an existing twin boundary. Cottrell's expression (1953) for the activation energy $\left(U_{c}\right)$ necessary to homogenously nucleate a twinning dislocation loop with a radius of $r_{c}$ is given by

$$
U_{c}=\frac{G B^{2} r_{c}}{4}\left[\ln \frac{r_{c}}{r_{0}}-1\right]
$$

where $G$ is the shear modulus, $b$ the magnitude of the Burgers vector and $r_{\mathrm{o}}=b / 4$. In tetragonal systems, the twin dislocation Burgers vector $b$ may be related to the lattice constants $(a$ and $c$ ) by the relation

$$
b=\left(c^{2}-a^{2}\right) /\left(c^{2}+a^{2}\right)^{1 / 2} .
$$


Since, the ageing behaviour (the degradation of properties with time) is known to depend upon the $c / a$ ratio of the material (McQuarrie and Bussem 1955) the magnitude of the dislocation Burgers vector might have an influence on the ageing behaviour of the material. Ageing is an important property of the ceramics for consideration when they are to be used in practical application. Although it is not seriously considered for commercial capacitor and transducer applications, it becomes quite important for high reliability applications such as high drive sonar ceramics and high quality PTC materials. It is known that ageing in these ceramics takes place due to the stress induced motion of non $180^{\circ}$ domains. The motion of these domains is influenced by various additives and hence by the composition of the body. The stress relaxation test gives scope to predict the ageing characieristics of different compositions.

Further, in the dislocation theory, the plastic strain rate is expressed as a power function of the shear stress as (Orowan $1940^{\circ}$, Johnston and Gilman 1959)

$$
\dot{\varepsilon}_{p}^{*}=\phi b N\left(\sigma^{*}\right)^{\mathrm{m}}
$$

where $\phi$ is a geometrical factor, $b$ the dislocation Burgers vector, $N$ the mobile dislocation density and $m$ the velocity stress exponent. Equations (1) and (9) permit estimating the velocity stress exponent $m$, a parameter which gives an index of the twin dislocation velocity in these materials. Thus, the stress relaxation technique offers the possibility of studying the kinetics of non $180^{\circ}$ domain walls in ferroelectric materials. In single crystals domain-wall kinetics were earlier studied (Bogdnov 1962) by optical observations using photographic techniques. Obviously such studies are cumbersome and timeconsuming. The stress relaxation method offers a more convenient method of study in such cases.

\section{Conclusion}

Stress relaxation studies on ferroelectric materials offer two important possibilities for study. (i) In polycrystalline ceramics, particularly in ceramics for use in professional and military applications, the stress relaxation test gives scope to predict the degradation characteristics or the ageing behaviour of the material. (ii) In small single crystals, it offers a convenient method of studying the kinetics of non $180^{\circ}$ domain walls under mechanical stress.

\section{Acknowledgement}

The author wishes to thank the management of Bharat Electronics for their encouragement to present this paper in the first conference on ferroelectrics, held at Delhi, December 1980 and also Prof Y V R K Prasad of the Indian Institute of Science, Bangalore, for helpful discussions.

\section{References}

Bogdnov S V 1962 Sov. Phys. Cryst. 7611

Cottrell A H 1963 Dislocations and plastic flow in crystals (Oxford: Clarendon Press) p 53

Esaklul K A, Gerberich W W and Koepke B G $1980 \mathrm{~J}$. Am. Ceram. Soc. 6325

Guiu F and Pratt P L 1964 Phys. Status Solidi 6111 
Jaffe $G$, Cuok $\mathrm{W} \mathrm{R}$ and Jaffe $\mathrm{H} 1971$ Piezolectric ceramics (London, Vew Yonk, Academu Press), 135

Johnston W G and Gilman J J. 1959 J. Appl. Phys. 30129

Lee D and Hart E W 1971 Mater. Trans 21245

Llyod D J and Embury J D 1971 Phys. Status Solidi 43393

MacEwen S R, Kupcis O A and Ramaswami B 1969 Scr. Metall. 3441

McQuarrie M C and Bussem W R 1955 Am. Ceram. Soc. Bull. 34402

Orowon E 1940 Proc. Phys. Soc. 528

Prasad V C S and Subbarao E C 1977 Ferroelectrces 15143

Prasad V C S and Subbarao E C 1973 Appl. Phys. Lett. 22424

Prasad V C S 1973 Stress induced motion of twin boundaries in $\mathrm{BaTiO}_{3}$ and $\mathrm{KNbO}_{3}$ single crystals $\mathrm{Ph} . \mathrm{D}$ Thesis Indian Institute of Technology, Kanpur

Prasad V C S and Subbarao E C 1972 Mater. Sci. Eng. 10297

Reed-Hill R E and Dahlberg E P 1966 Trans. AIME 236679

Rhodes R C 1951 Acta Crystallogr. 4105

Subbarao E C, McQuarrie M C and Bussem W R 1957 J. Appl. Phys. 281194

Syrkin L N and El'gard A M 1965 Sov. Phys. Soltd State 7967

Wood E A 1951 Acta Crystallogr. 4353 